\title{
Ictal EEG in sunflower syndrome: Provoked or unprovoked seizures?
}

\author{
Jo Sourbron ${ }^{\mathrm{a}}$, Neishay Ayub ${ }^{\mathrm{b}}$, Yancheng Luo ${ }^{\mathrm{c}}$, Elizabeth A. Thiele ${ }^{\mathrm{c}, *}$ \\ a Department of Development and Regeneration, Section Pediatric Neurology, University Hospital KU Leuven, Leuven, Belgium \\ b Clinical Neurophysiology and Epilepsy Program, Department of Neurology, Massachusetts General Hospital, Boston, MA, USA \\ ${ }^{c}$ Pediatric Epilepsy Program, Department of Neurology, Massachusetts General Hospital, Boston, MA, USA
}

\section{A R T I C L E I N F O}

\section{Article history:}

Received 28 June 2020

Revised 27 August 2020

Accepted 30 August 2020

Available online $\mathrm{xxxx}$

\section{Keywords:}

Epilepsy

Sunflower syndrome

Clinical series

Ictal EEG

\begin{abstract}
A B S T R A C T
Sunflower syndrome (SS) is a rare, photosensitive epilepsy characterized by an attraction to light and highly stereotyped seizures with associated hand-waving (HW). It is controversial whether $\mathrm{HW}$ is part of the seizure or a provoking factor; therefore, we aimed to characterize the ictal electroencephalogram (EEG) in patients with SS. Video-EEG (vEEG) and charts of five patients with SS with HW during vEEG from Massachusetts General Hospital's Pediatric Epilepsy Program were reviewed and analyzed.

In four out of five patients, the ictal EEG showed high amplitude (500-700 $\mu \mathrm{V}) 3-4 \mathrm{~Hz}$ generalized spike/ polyspike-and-slow wave discharges, lasting 1.63-24.41 s.

One hundred and twelve of $126 \mathrm{HW}$ episodes, correlating to epileptiform activity (vEEG), had a lag time of less than $1.00 \mathrm{~s}(88.89 \%)$ between onset of HW and appearance of epileptiform activity. This suggests that HW does not induce seizure activity.

Awareness of the ictal EEG features of this syndrome is important, as patients are frequently described as "selfinducing" their seizures.
\end{abstract}

(c) 2020 Elsevier Inc. All rights reserved.

\section{Introduction}

Sunflower syndrome (SS) is a photosensitive, often drug-resistant, epilepsy syndrome characterized by highly stereotyped reflex seizures and compulsions to seek out and turn towards light, similar to a "sunflower turning towards the sun" [1,2]. The seizures are characterized by episodes of hand-waving (HW) in front of the eyes, often accompanied by eye fluttering and/or rapid lateral head rotation and may or may not impair consciousness [3,4]. Often, SS is initially misdiagnosed as a tic or behavioral disorder due to the stereotyped abnormal movements and light-seeking behavior. A correct diagnosis is important to implement proper treatment and avoid a negative emotional impact on the patient $[2,5]$.

The first report of SS is likely by H. Gastaut in 1951 who described two children that seemed to seek out the light and induce seizures by rapidly passing their hands in front of their eyes (HW) [6]. In 1962, Andermann et al. reviewed 34 photosensitive epilepsies of which 21 patients had self-induced seizures and 11 showed HW episodes in the sun [7]. Two recent case reports [3,8] and a case series [9] also suggest that

* Corresponding author at: Massachusetts General Hospital, 175 Cambridge Street, Suite 340, Boston, MA 02114, USA.

E-mail address: ETHIELE@mgh.harvard.edu (E.A. Thiele). the seizures in SS are self-induced by HW. It has also been suggested that HW episodes may be a part of the seizure, not the provoking trigger $[1,10,11]$.

Electroencephalogram (EEG) features include ictal 3-4 Hz generalized spike and wave (GSW) discharges, suggestive of an underlying generalized epilepsy [3,11].

The goal of this study was to further characterize the ictal EEG in SS and to determine if HW is part of the seizure or a trigger for seizure.

\section{Materials and methods}

\subsection{Patients}

We reviewed the medical records of patients with the diagnosis of SS seen at Massachusetts General Hospital (MGH), in Boston, USA. Approval from the Partners Human Research Committee was obtained prior to this study.

The diagnosis of SS was made based on clinical features including photosensitivity and attraction to bright and/or artificial lights in patients that have seizures involving $\mathrm{HW}$, often accompanied by head shaking, eye rolling/fluttering $[1,2,9,11]$.

We characterized demographics including family history, age at onset, prior symptoms, seizure types, seizure frequency, treatment history, and analyzed available video-EEG (vEEG) recordings. 


\subsection{Ictal EEG}

The vEEG were analyzed by two neurologists (NA, YL) and researcher (JS). Continuous EEG/CCTV monitoring was performed using the standard 10-20 electrode placement in the Epilepsy Monitoring Unit (EMU), which are standardized hospital rooms with a window. Patient E, however, had vEEG in a windowless room (in the EEG lab). Standard referential and bipolar montages were used for EEG review, as well as specifically reformatted arrays when necessary. A push-button was available for the patient and their patient's family and/or staff to flag the occurrence of HW episodes. Only the push-buttons episodes were evaluated. Continuous computerized spike and seizure detection was performed throughout the recording period.

The start of the HW episode was defined as when the hand (first digit) was initially in front of the patient's face. This was always documented and verified by NA and JS. The end of the HW episode was defined as the time the hand last went in front of the face. HW episodes were excluded if no definite start could be identified. The start of the spike and wave activity was defined as the first spike/sharp and the end as the end of the final slow wave. The predominant region was defined as the EEG region with the highest amplitude.

Parameters evaluated included number of episodes, duration, epileptiform activity coincident/following HW, lag time (time from start of HW until epileptiform activity), duration of epileptiform activity, and predominant region.

\subsection{Graphs and figures}

GraphPad Prism 6 software (GraphPad Software, Inc.) was used for graphical visualizations. Electroencephalogram readouts were visualized by Natus.

\section{Results}

\subsection{Patients}

Eight patients with SS had undergone vEEG monitoring. Of these eight patients, two had no HW episodes during the study, and one had episodes only while off camera; therefore, these patients were excluded from our analysis. Thus, the medical records of five patients (patients A, B, C, D and E; all female) were reviewed (Table S1, supplementary data).
All of these patients were attracted to bright and/or artificial light. None of these patients had intellectual disability (ID). Only one patient (B) had genetic testing (epilepsy gene panel), which did not reveal any pathogenic mutation. In all five patients, intermittent photic stimulation (IPS) was performed, and in three out of five patients, (60\%) there was a clear photoparoxysmal response (PPR) (supplementary data, Table S2 and Fig. S3).

\subsection{Ictal EEG}

Four of the five had undergone $24 \mathrm{~h}$ vEEG monitoring. For logistical reasons, patient $\mathrm{E}$ had $3 \mathrm{~h}$ and 20 min of recording, during which 30 HW episodes were captured. During HW episodes, there were no other seizure types reported, although other seizure types (atonic and myoclonic seizures) have been described in some patients (Table S1). All of the patients spreadout their fingers during HW, and none of them rubbed their eyebrows.

One patient (patient $\mathrm{D}$ ) did not have any definite epileptiform activity in the EEG during HW, although the ictal EEG showed a sharply contoured background with amplitudes of $80-200 \mu \mathrm{V}$ during the HW episodes, which was not seen at other times during the EEG. In patients $\mathrm{A}, \mathrm{B}, \mathrm{C}$, and E, the HW ictal vEEG was characterized by high amplitude (500-700 $\mu \mathrm{V}$ ) 3-4 Hz spike/polyspike-and-slow wave discharges (Fig. 1 and Table S2).

Five patients had HW episodes that appeared to be induced by bright sunlight filtering through large windows or by indoor lighting (patient E). All had stereotyped HW, characterized by waving one of their hands in front of their eyes, sometimes with a slight angle and palmar side towards the face. The HW episodes were typically clustered with brief pauses between the episodes. All patients waved the same hand during all of the HW episodes; this was the dominant hand in three out of five patients (Table S2). There were no HW episodes without a bright light present and none during sleep.

The majority of HW episodes, 63.85\% (mean; standard deviation $(S D)=33.72$ ), was associated with epileptiform activity (Fig. S2A). If patient $\mathrm{D}$ was excluded from analysis, the percentage was higher ( mean $=80.01$; SD = 11.96). Overall, HW episodes lasted from $1.63 \mathrm{~s}$ (patient B) to $24.41 \mathrm{~s}$ (patient D) (Fig. S2B).

For all, with the exception of patient D, HW was associated with GSW or generalized polyspike and wave activity on EEG. The lag time, i.e., the time between the start of HW and the start of the epileptiform
A

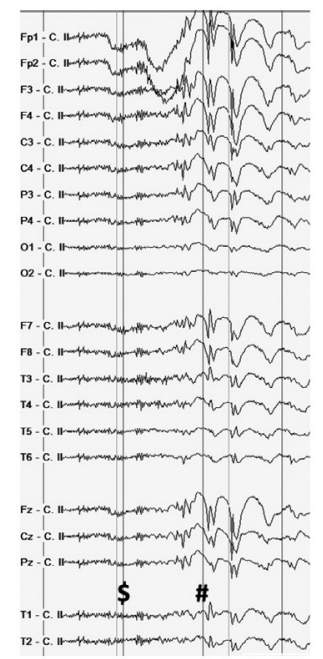

\section{B}

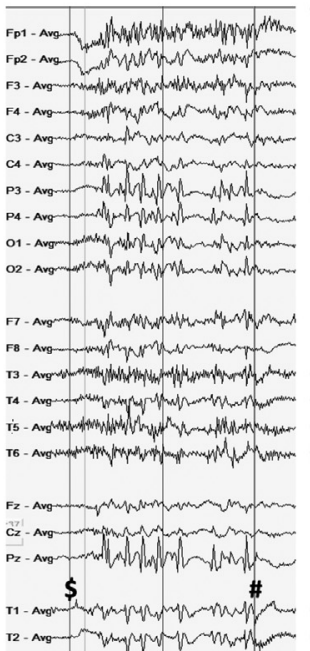

C

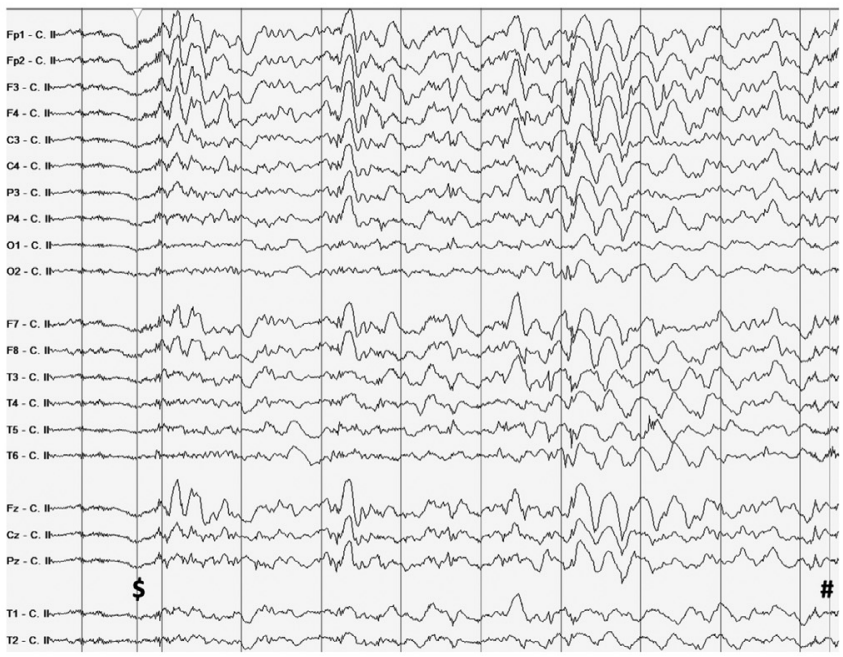

$\mathrm{E}$

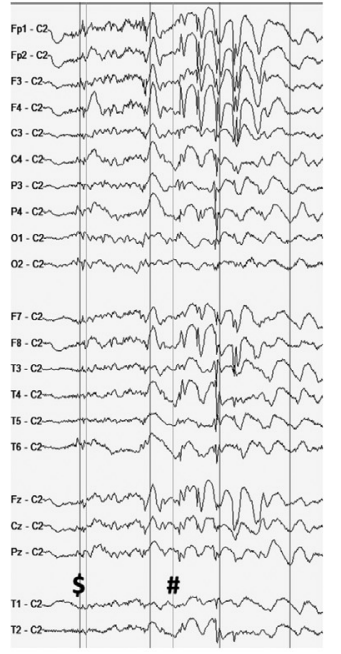

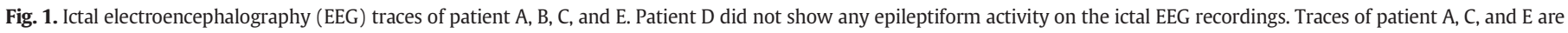

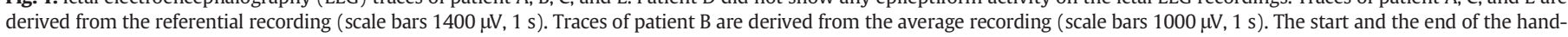
waving (HW) episode is depicted by an $\$$ and \#, respectively. 


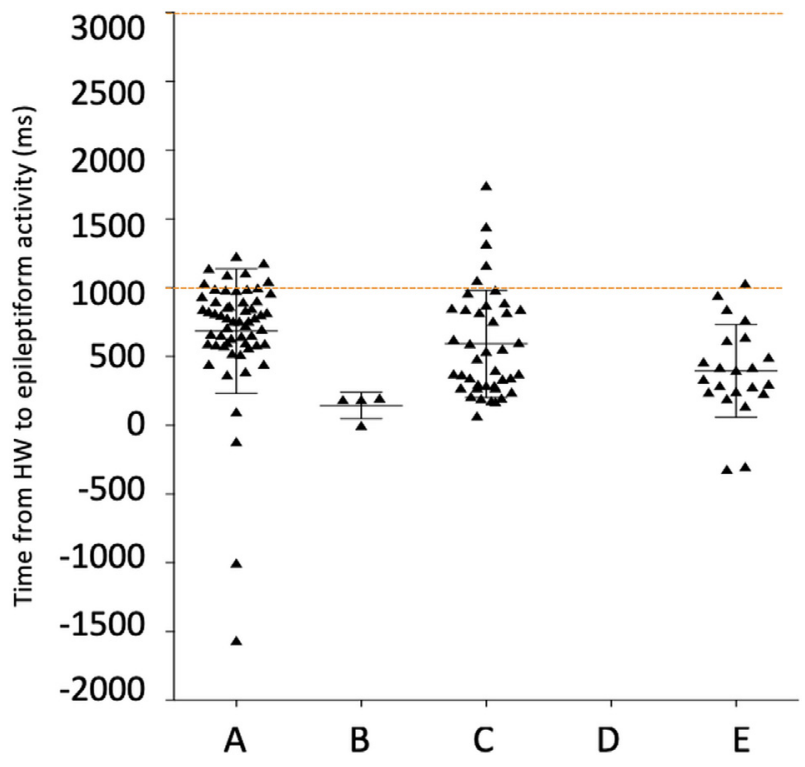

Fig. 2. Time from hand-waving (HW) to epileptiform activity, generalized spike and wave, or generalized polyspike and wave activity (lag time), analyzed for four out of five patients (A, B, C, and E). Patient D did not show any epileptiform activity on the EEG recordings. Most episodes correlated to epileptiform activity on the EEG had a lag time below $1.00 \mathrm{~s}$, $1000 \mathrm{~ms}$ (lowest, horizontal orange line). (For interpretation of the references to color in this figure legend, the reader is referred to the web version of this article.)

activity on the EEG was measured for patients A, B, C, and E. Of the 126 HW episodes that were associated with epileptiform activity on the EEG, 112 (88.89\%) had a lag time below 1.00 s (Fig. 2).

\section{Discussion}

Although it has been speculated that HW episodes induce the seizure activity, this is not consistent throughout the literature $[3,8,11]$. Some reports have shown that the seizure onset occurs at the same time as the HW, suggesting that HW may be part of the seizure rather than the provoking factor $[1,2,10,11]$.

Our results from analyzing vEEG recording of HW episodes underline the hypothesis that the HW does not induce the abnormal activity on the EEG but may be part of the ictal semiology. Supporting this, $88.89 \%$ of the HW episode-associated epileptiform activity on the EEG had a lag time below $1.00 \mathrm{~s}$. Wolf et al. describes that for reflex epilepsies, simple seizure triggers are sensory (mostly visual stimulus) and can act within seconds [12]. To be defined as stimulus-evoked epileptic seizures, the lag time from a stimulus to the seizure onset has been proposed to be between 1.00 and $3.00 \mathrm{~s}$ [13]. Regarding the HW episodes (potential stimulus) and epileptiform activity, the majority had a lag time below $1.00 \mathrm{~s}$, thus likely not provoking the seizure. In contrast, intermittent photic stimulation (IPS) of less than $1.00 \mathrm{~s}$ generated epileptiform activity (PPR) in three patients of our cohort; these were not associated with HW episodes (supplementary data). Thus, our data suggest that HW episodes are possibly part of the ictal event, although epileptiform activity (PPR) can be provoked by visual stimuli such as IPS.

Over $80 \%$ of all HW episodes in patients A, B, C, and E were clearly related to epileptiform activity with a duration of $1.63 \mathrm{~s}$ up to $24.41 \mathrm{~s}$. The HW episodes during vEEG were all limited to one hand (left or right) and involved the dominant hand in $60 \%$ of the patients (Table S2).

The 3-4 Hz GSW ictal activity seen in these patients with SS is similar to other childhood-onset generalized epilepsies, such as childhood absence and juvenile myoclonic epilepsy (JME) [3,11], suggesting a possible relationship.

Although we analyzed a large number of HW episodes, the number of patients involved in the study was small. We did not quantitate aspects of being drawn to or going to the light, so it cannot be fully excluded that compulsive behaviors in patients with SS (going to the light and starting the HW) might induce seizures [13]. The vEEG of one patient (patient $\mathrm{D}$ ) did not show any definite epileptiform activity during HW, although the ictal EEG had different consistent features that were different than her interictal EEG, and her HW episodes had the longest duration (Fig. S2).

\section{Conclusions}

Our findings agree with prior studies that propose that the seizures in patients with SS are not self-induced $[1,2,10,11]$ but rather that HW may be part of the ictal event. Further research is needed to better understand the relationship between HW episodes and seizures in patients with SS. Physicians should be aware of this epilepsy syndrome and that the HW episodes may not be volitional as a trigger to "self-induce" seizures in order to avoid potentially increasing the psychological burden and stigma of the disease.

Additional research is needed to further characterize SS, including better understanding the natural history and potential genetic etiology of the disorder. This would improve the scientific understanding and hopefully treatment of this complex epilepsy syndrome.

\section{Declaration of competing interest}

Elizabeth A. Thiele, M.D., Ph.D. has served as a paid consultant for GW Pharma, Zogenix, Biocodex, West Therapeutics, Aquestive and Regenxbio. The remaining authors have no conflict of interest to disclose. We confirm that we have read the Journal's position on issues involved in ethical publication and affirm that this report is consistent with those guidelines.

\section{Acknowledgments}

The authors of this paper would like to thank the patients and their families for cooperating in the study, and for allowing their data to be used.

\section{Appendix A. Supplementary data}

Supplementary data to this article can be found online at https://doi. org/10.1016/j.yebeh.2020.107470.

\section{References}

[1] Ames FR. "Self-induction" in photosensitive epilepsy. Brain. 1971;94:781-98. https://doi.org/10.1093/brain/94.4.781.

[2] Barnett JR, Fleming BM, Geenen KR, Sourbron J, Freedman JH, Bruno PL, et al. Characterizing sunflower syndrome: a clinical series. Epileptic Disord. 2020. https://doi. org/10.1684/epd.2020.1161.

[3] Belcastro V, Striano P. Self-induction seizures in sunflower epilepsy: a video-EEG report. Epileptic Disord. 2014;16:93-5. https://doi.org/10.1684/epd.2014.0630.

[4] Aicardi J, Gastaut H. Treatment of self-induced photosensitive epilepsy with fenfluramine. N Engl J Med. 1985;313:1419. https://doi.org/10.1056/NEJM198511283132218.

[5] Sethi NK, Labar D, Torgovnick J. Myoclonic epilepsy masquerading as a tic disorder. Clin Neurol Neurosurg. 2007;109:509-11. https://doi.org/10.1016/j.clineuro.2007.02.013.

[6] Gastaut H. L'épilepsie photogénique Rev Praticien ; 1951.

[7] Andermann K, Berman S, Cooke PM, Dickson J, Gastaut H, Kennedy A, et al. Self-induced epilepsy. A collection of self-induced epilepsy cases compared with some other photoconvulsive cases. Arch Neurol. 1962;6:49-65.

[8] Singhi PD, Bansal D. Self-induced photosensitive epilepsy. Indian J Pediatr. 2004;71: 649-51. https://doi.org/10.1007/bf02724128.

[9] Baumer FM, Porter BE. Clinical and electrographic features of sunflower syndrome. Epilepsy Res. 2018;142:58-63. https://doi.org/10.1016/j.eplepsyres.2018.03.002.

[10] Livingston S, Torres IC. Photic epilepsy: report of an unusual CASE and review of the literature. Clin Pediatr (Phila). 1964;3:304-7. https://doi.org/10.1177/ 000992286400300511.

[11] Ames FR, Saffer D. The sunflower syndrome. A new look at "self-induced" photosensitive epilepsy. J Neurol Sci. 1983;59:1-11.

[12] Wolf P. Reflex epileptic mechanisms in humans: lessons about natural ictogenesis. Epilepsy Behav. 2017;71:118-23. https://doi.org/10.1016/j.yebeh.2015.01.009.

[13] Panayiotopoulos CP. The epilepsies: seizures, syndromes and management. Oxfordshire (UK): Bladon Medical Publishing; 2005. 